\title{
Validity and Reliability Determination of the Persian Version of Prosthesis Evaluation in Individuals with Lower Limb Amputations Questionnaire
}

\section{ART ICLE IN F O}

\section{Article Type}

Descriptive Study

\section{Authors}

Adel Gomnam M. ${ }^{1} B S C$

Kamali M.* $P h D$,

Mobaraki H. ${ }^{1} P h D$,

Saeedi $H^{2} P h D$

How to cite this article
Adel Gomnam M, Kamali M,
Mobaraki H, Saeedi H. Validity
and Reliability Determination of
the Persian Version of Prosthesis
Evaluation in Individuals with
Lower Limb Amputations
Questionnaire. Iranian Journal
of War \& Public Health.
2016;8(1):9-16

\section{A B S T R A C T}

Aims Post-amputation rehabilitation can improve the quality of life. Prosthesis evaluation questionnaire (PEQ) has been designed to evaluate the prosthetic functioning and major aspects of the quality of life in persons with lower-limb amputations. The aim of this study was to translate the questionnaire into Persian and evaluate validity and reliability of the translation. Instrument \& Methods The study was done qualitatively and quantitatively. Firstly, prosthesis evaluation questionnaire was translated into Persian and localized. To evaluate formal validity, the questionnaire was completed by 17 lower-limb amputees referred to Kowsar orthotics and prosthesis center. Its content validity was evaluated by 15 prosthesis experts. Using test-retest method, the questionnaire was completed by 70 amputees referred to the center in 2015 . The amputees were selected by simple non-probability method. The questionnaire was completed by 22 of the amputees after 2 weeks, again. Data was analyzed using Cronbach's alpha, relative repetition coefficient, and impact score.

Findings The impact score of all the items of the questionnaire was more than 1.5 and favorable. Excluding formal scale and relative repetition coefficient, Cronbach's alpha was more than the favorable level (0.7) in other scales.

Conclusion Validity and reliability of the Persian version of prosthesis evaluation questionnaire are favorable; and the version can be used to assess prosthesis and major aspects of the quality of life in the Iranian amputees.

Keywords Reproducibility of Results; Amputation; Prosthesesand Implants; Iran
*Rehabilitation Management Department, Rehabilitation Faculty, Iran University of Medical Sciences, Tehran, Iran

${ }^{1}$ Rehabilitation Management Department, Rehabilitation Faculty, Iran University of Medical Sciences, Tehran, Iran

${ }^{2}$ Orthosis \& Prosthesis Department, Rehabilitation Faculty, Iran University of Medical Sciences, Tehran, Iran

\section{Correspondence}

Address: Rehabilitation Faculty, Madad Karan Street, Shahid Shah Nazari Street, Madar Square, Mirdamad Boulevard, Tehran, Iran Phone: +982122221577

Fax: +982122220946

kamali@mkamali.com

\section{Article History}

Received: January 7, 2016

Accepted: March 9, 2016

ePublished: April 3, 2016

\section{CITATION LINKS}

1] Atlas of limb prosthetics: Surgical, prosthetic, and ... [2] Maintaining support in people with ... [3] Orthotics and prosthetics in ... [4] Iranian casualties during the eight years of IraqIran ... [5] Quality of life: The assessment, analysis and interpretation of patient-reported ... [6] The value of adding the Quality of Life Enjoyment and Satisfaction Questionnaire to outcome assessments of psychiatric inpatients with mood and affective ... [7] Musculoskeletal pain and overuse syndromes in adult acquired major upper-limb ... [8] Depression and incident lower limb amputations in ... [9] Amputees in limburg: Incidence, morbidity and mortality, prosthetic supply, care utilisation and functional level ... [10] Prosthetics research study ... [11] Scientific and clinical problems in indexes of functional ... [12] Functional assessment measures in ... [13] Prosthesis evaluation questionnaire for persons with lower limb ... [14] Lower limb prosthetic outcome measures: a review of the ... [15] A systematic review of functional and quality of life assessment after major lower ... [16] Use of the prosthesis evaluation ... [17] Guidelines for the process of cross-cultural adaptation of ... [18] Prosthesisrelated QOL of the people with amputation in ... [19] Comparison of prosthetic outcomes between adolescent transtibial and ... [20] Measuring long-term outcome in people with lower limb ... [21] Measuring mobility in people with lower limb amputation: Rasch analysis of ... [22] Cross-cultural validation of the ... [23] Cross cultural equivalence testing of the ... [24] The prosthesis evaluation questionnaire: Reliability and cross-validation of ... [25] Translating health status questionnaires and ... [26] Research Methods in ... [27] Measurement in medicine: a practical ... [28] Quantifying test-retest reliability using the intraclass correlation coefficient and ... [29] Cronbach's alpha: A tool for assessing ... [30 How to choose outcomes relevant to the client and ... [31] The short form health survey ... 
مقدمه - - مق

قطع عضو يكى از شايعترين معلوليتهاى حركتى است و مسالهاى

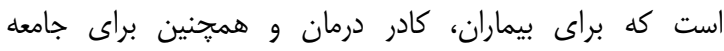

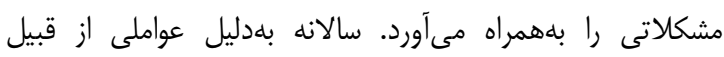

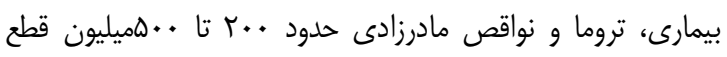

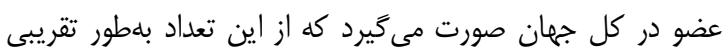

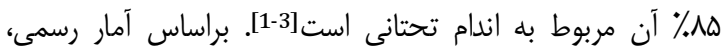

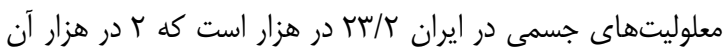

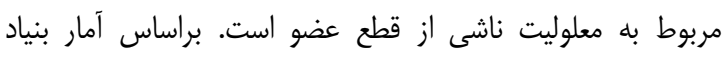

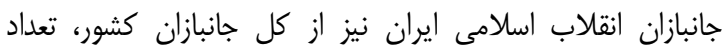

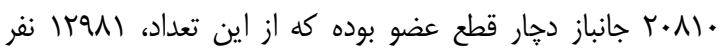

$$
\text { مبتلا به قطع عضو اندام تحتانى هستند [4]. }
$$

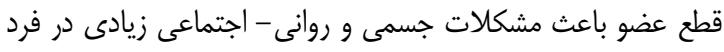

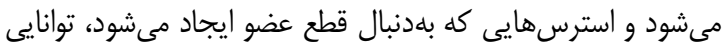

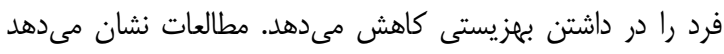

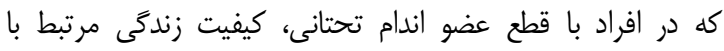

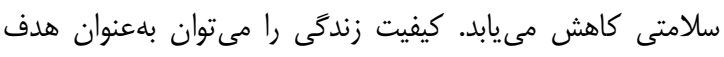

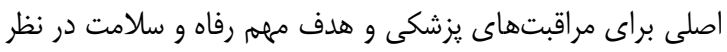

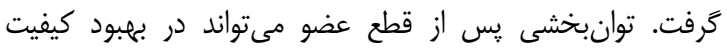

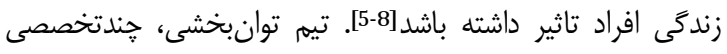

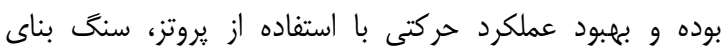

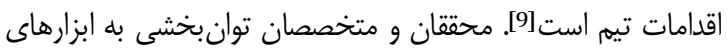
اندازمكيرى براى تعيين تغيير در وضعيت عملكردى، در نتيجه

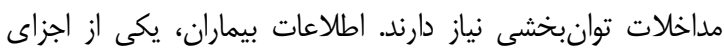

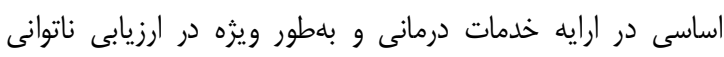
عملكردى و كيفيت زندگى مرتبط با سلامتى است [12-12].

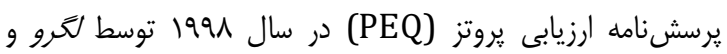

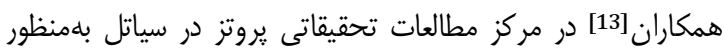

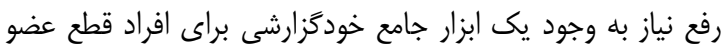

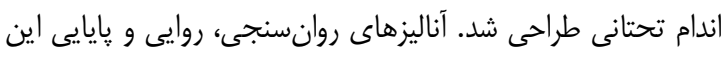

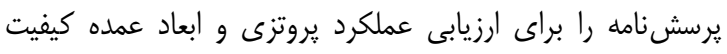

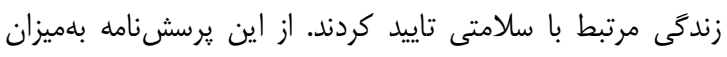

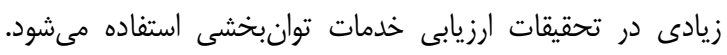

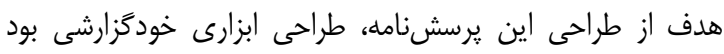

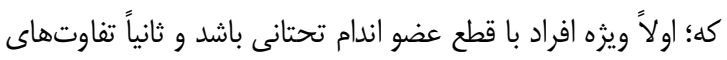

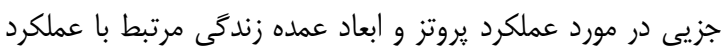
يروتز را اندازه بخيرد[10,13].

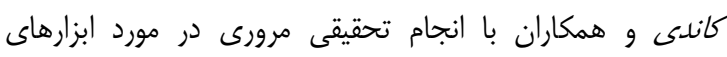

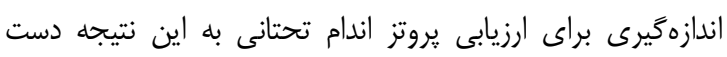

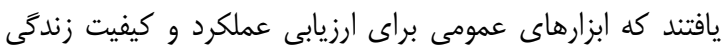

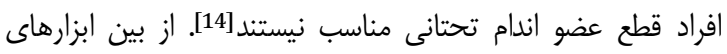

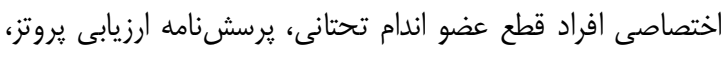

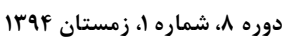

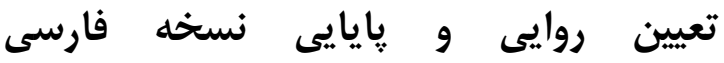 يرسشنامه ارزيابى :روتز افراد داراى قطع عضو اندام تحتانى}

MSc مريم عادل تمنام مدون

كروه مديريت توانبخشى، دانشكده توانبخشى، دانشعاه علوم يزشكى ايرى ايران، تهران، ايران

PhD محمد كمالى

كروره مديريت توانبشى، دانشكده توانبشى، دانشكاه علوم يزشكى ايران، تهران، ايران

حسين مباركى PhD

كروه مديريت توانبخى، دانشكده توانبخشى، دانشًاه علوم يزشكى ايران، تهران، ايران

حسن سعيدى PhD

كروه اعضاى مصنوعى، دانشكده توانبخشى، دانشعاه علوم يزشكى ايران، تهران، ايران

جִكيده

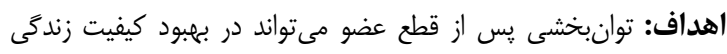

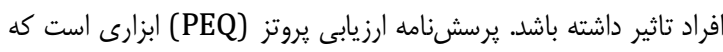

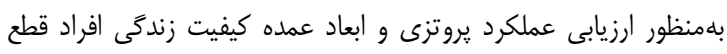

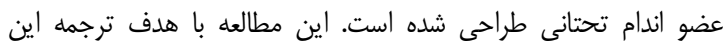

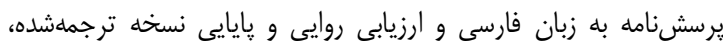

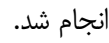

ابزار و روشهام شلد: اين بزوهش به دو روش كيفى و كمى انجام شد. ابتدا

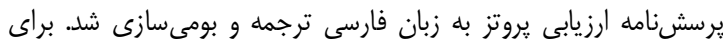

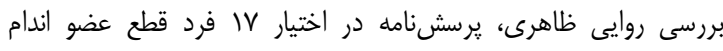

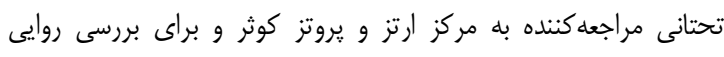

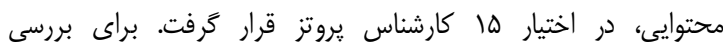

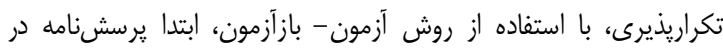

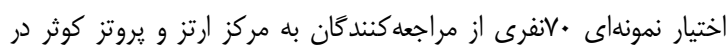

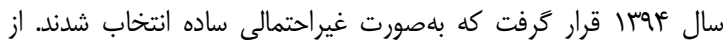

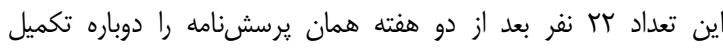

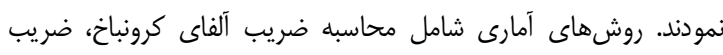

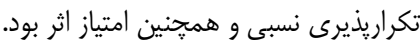

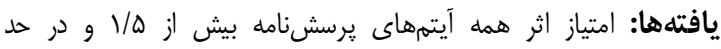

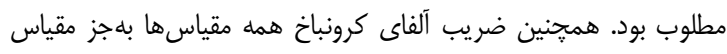

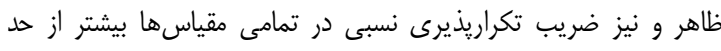
مطلوب / م • بهدست آمد.

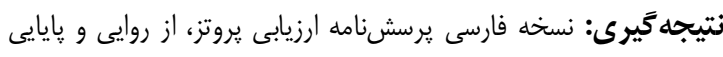

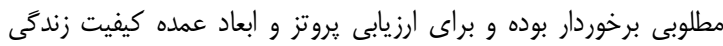
افراد قطع عضو فارسىزبان قابل استفاده است.

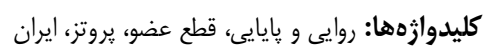

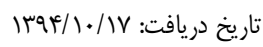
تاريخ يذيرش: تانئ: "نويسنده مسئول: kamali@mkamali.com 


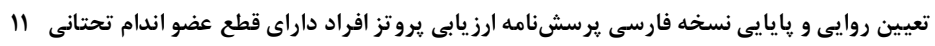

استفاده در كلينيكهاى كشورهاى مختلف به زبان رسمان رسمى آن آن كشور

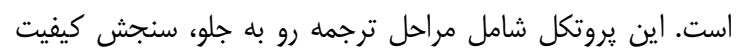

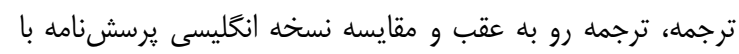

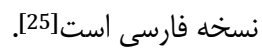

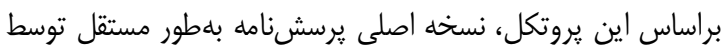

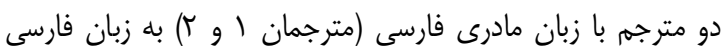

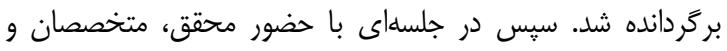
مترجمان، با بحث روى تفاوت بين ترجمهها و مستندسازى آنها،

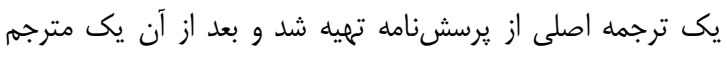

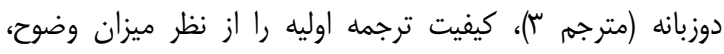

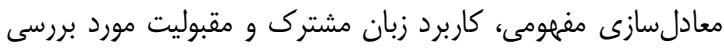

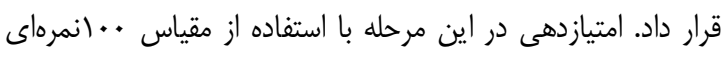

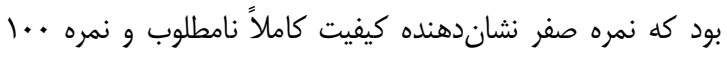

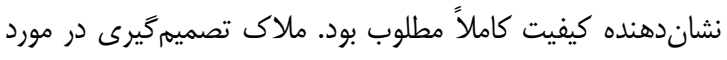
كيفيت ترجمه سئوالات، نمره كيفيت يايينتر از •م براى كيفيت

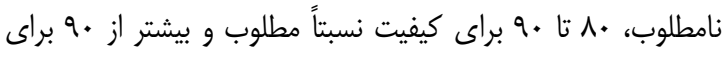
ترجمه مطلوب بود. ترجمه اوليه توسط دو مترجم ديخر با زبان

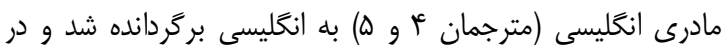
آخر نسخه نهايى توسط كميته كارشناسى شامل مترجمان، تيم

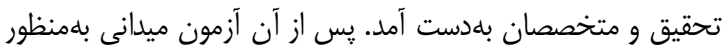

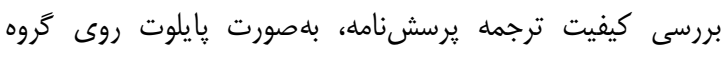

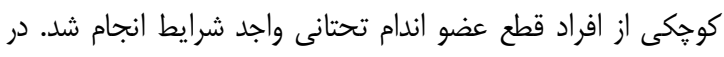

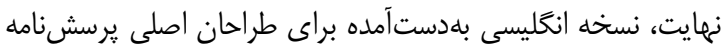

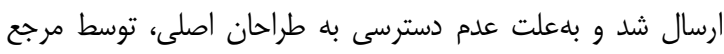
معتبر در آن زمينه، از لحاظ مفهوم، مورد بررسى و و تاييد قرار

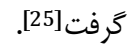

نسخه اصلى PEQ كه يرسشنامه خودَزارشى است از بر سئوال و

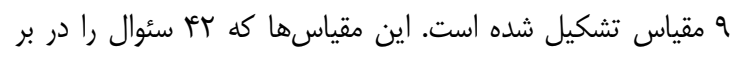

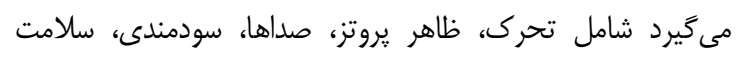

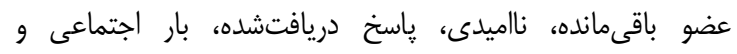

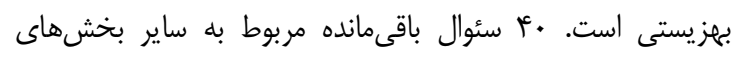

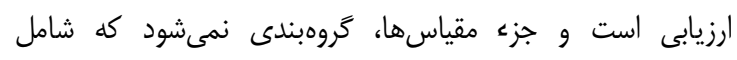

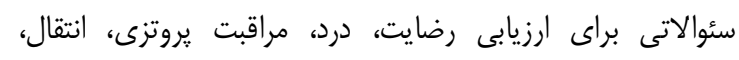

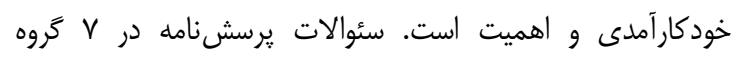

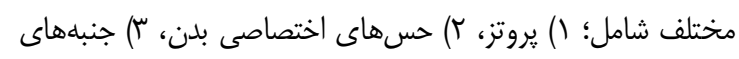

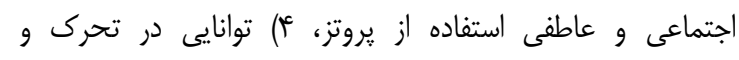

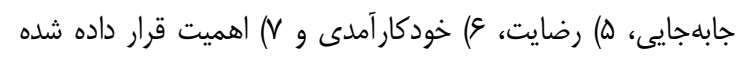

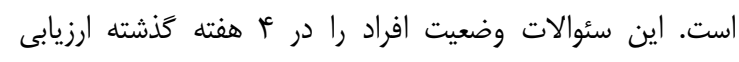

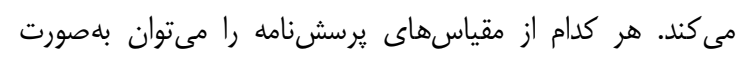

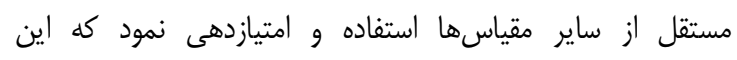
انعطافيذيرى براى محققان فرصتى را فراهم مى مند تا روى مقئي محتواى مقياسهاى مورد نظر از يرسشنامه تمركز كنند و تنها بـ ميه

Scholarly Quarterly of Iranian Journal of War and Public Health

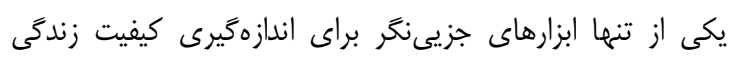

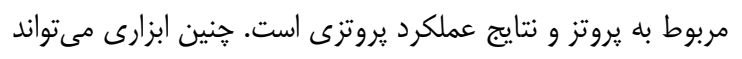

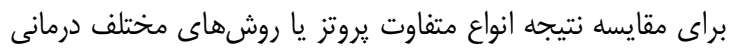

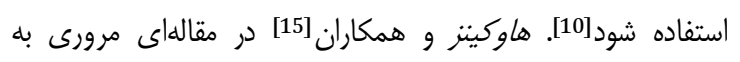

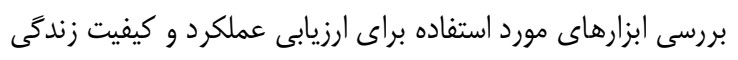

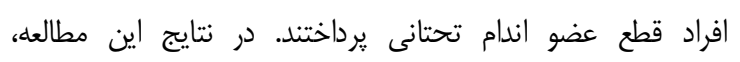
بالاترين امتياز در ميان ابزارهاى اختصاصى كيفيت زندكى إنى افراد

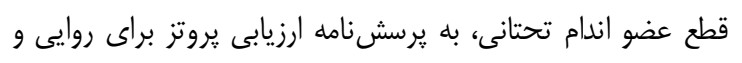

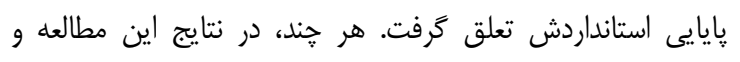

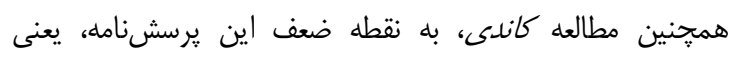

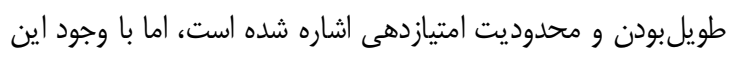
ضعف، وجود انعطاف در استفاده از هر كدام از مقياسهاى إنى اين

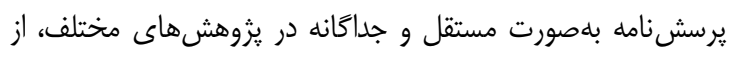
مزاياى آن محسوب مىشود [10,16].

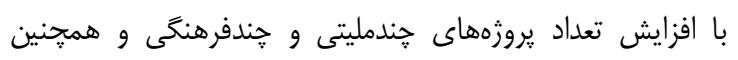

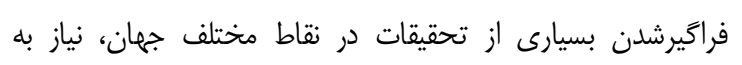

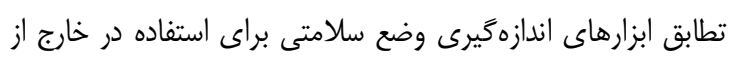

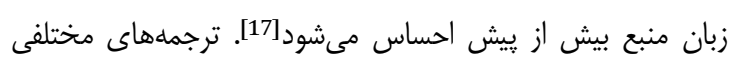
كه از اين يرسشنامه انجام شده شامل فرانسوى، آلمانى [16]،

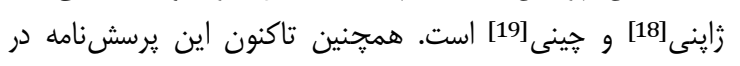

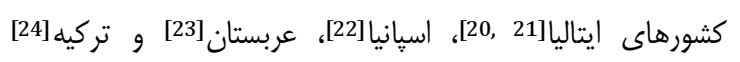
اعتبارسنجى شده و تغييرات فرهنكى لازم با توجه به مسايل قومى إنى

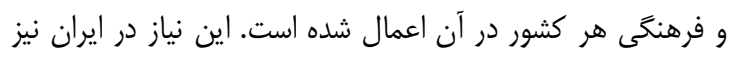
احساس مىشد تا بتوان نسخه فارسى يرسشنامه ارزيابى يروتز

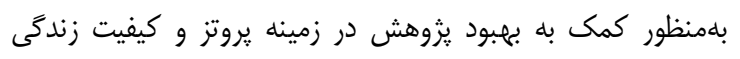

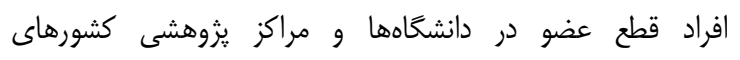
فارسىزبان (ايران، افغانستان و تاجيكستان) استفاده نمود.

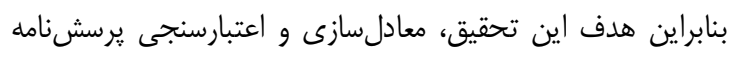

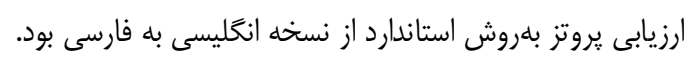

\section{ابزار و روشها}

بلمنظور ترجمه و روانسنجى يرسشنامه ارزيابى يروتز از روشهاى رون كيفى و كمّى بلهورت زير استفاده شد: ترجمه: ابتدا فرم بررسى ڤيرسش نامه ارزيابى یروتز (PEQ) تكميل

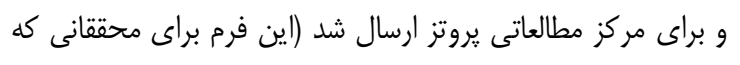

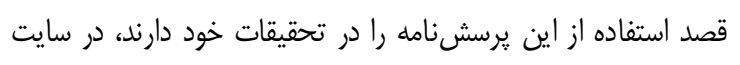

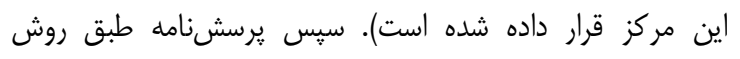

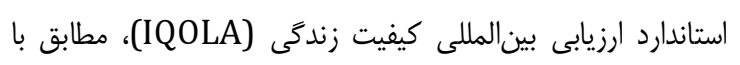

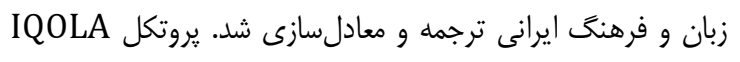
در سال 1991 توسط سازمان جهانى بهداشت تصويب شد كه هدف

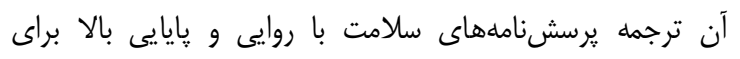


بلمنظور جمع آورى دادهها، برسشنامه ارزيابى تروتز بههمراه فرم

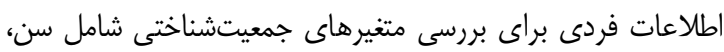

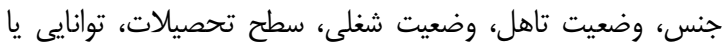
عدم توانايى رانندگى بهعلت مشكلات بروتزى، سطح قطع عضو (بالاى زانو، روى زانو، زير زانو و قطع عضو مفصل مج)، علت قطع مانع

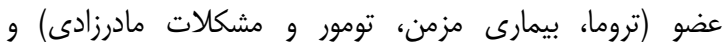

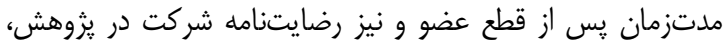

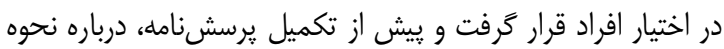

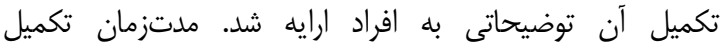

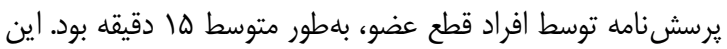

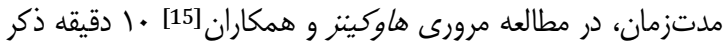

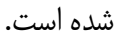
براى بررسى تكراريذيرى از روش بايايى آزمون- بازآزمون استفاده

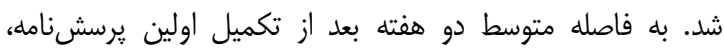

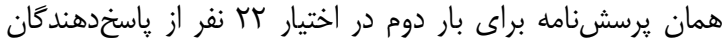

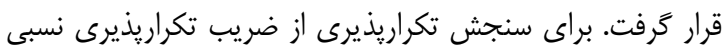

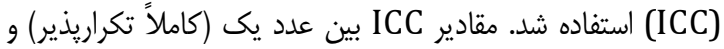

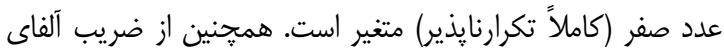

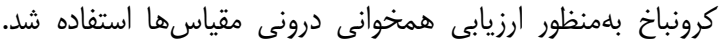

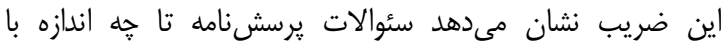

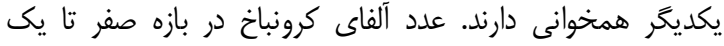

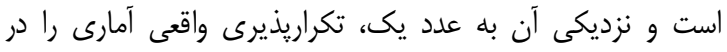

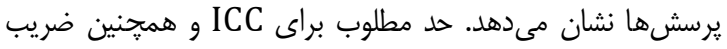

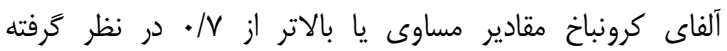

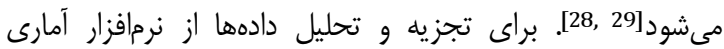

SPSS 21

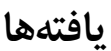

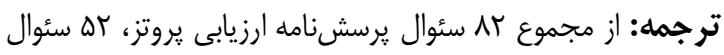

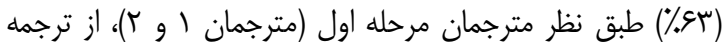

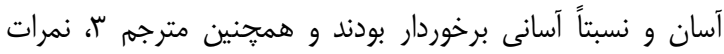

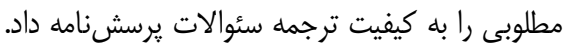

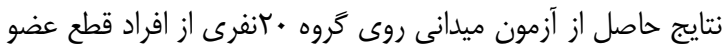

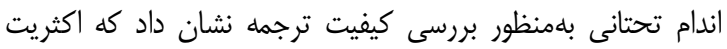

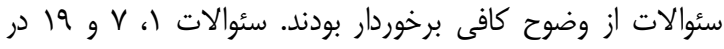

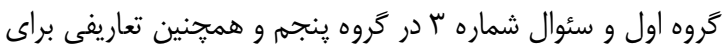

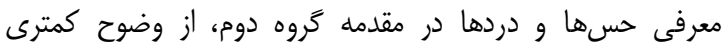

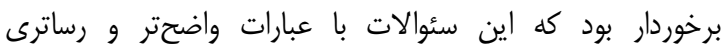

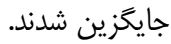
روايى: نمره اثر بهدستآمده براى روايى ظاهرى يرسشنامنامه، براى

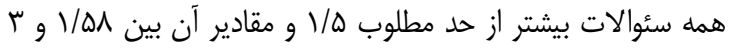

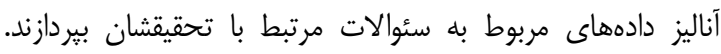

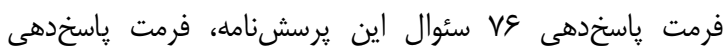

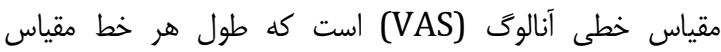

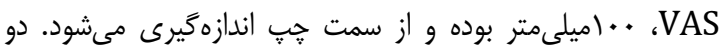

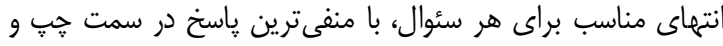

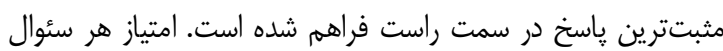
با اندازمكيرى فاصله محل تقاطع خط مورد نظر فرد قطع عضو بال

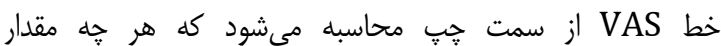

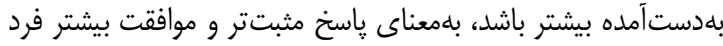
با آن سئوال است. امتياز هر مقياس نيز، با محاسبه ميانكَين امتياز سئوالات مربوط به مقياس بهدست مى آيد و براى محاسبه ميانخين، لازم است كه ياسخدهنده به حداقل نيمى از سئوالات هر مقياس بلاس

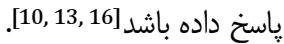
روايى: بررسى روايى يرسشنامه طى دو مرحله روايى ظاهرى و إسدا

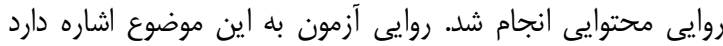

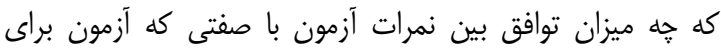

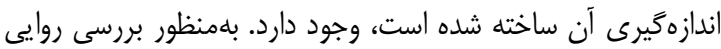

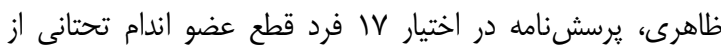

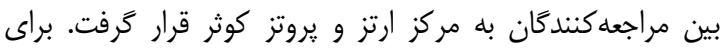

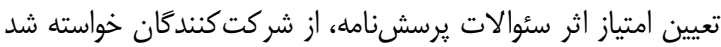

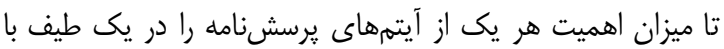

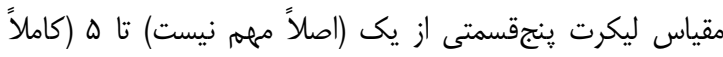

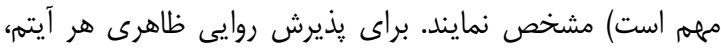

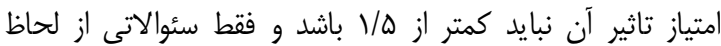

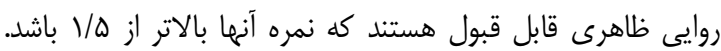

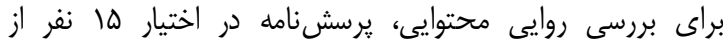

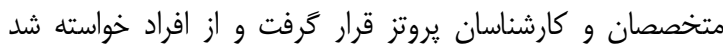

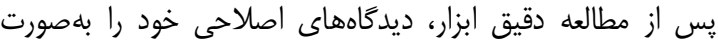
مبسوط و كتبى ارايه نمايند[26,27]. يايايى: جامعه آمارى يثوهش را إ افراد قطع عضو اندام تحتانى

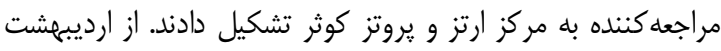

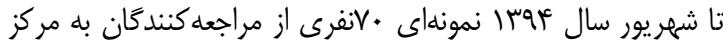

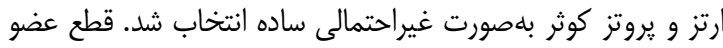
يكسرفه سطح مفصل مج يا بالاتر، داشتن سواد خواندن و نوشتن،

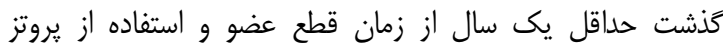
حداقل بلمدات ه روز در هفته از معيارهاى ورود به مطالعه بود [13].

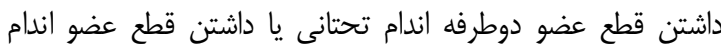
فوقانى، داشتن اختلالات روانى يا ساير مشكالاتى كه مانع استفاده

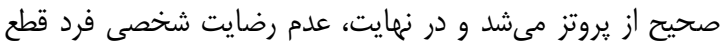

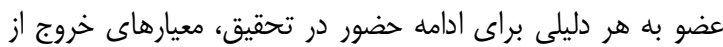

مطالعه بودند [13, 24. 


\section{بحث}

اين مطالعه با هدف ترجمه و روانسنجى يرسشنامه ارزيابى يروتز

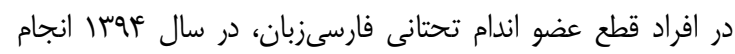

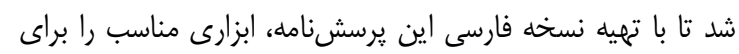

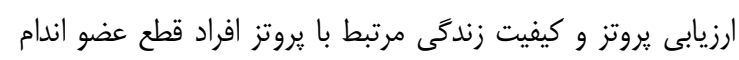

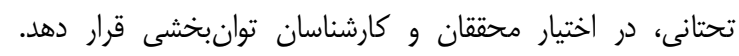

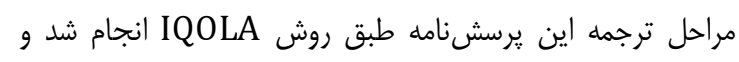

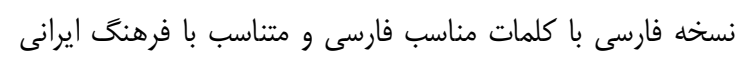

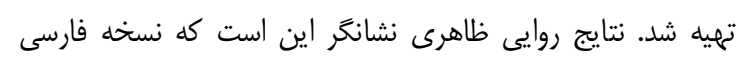

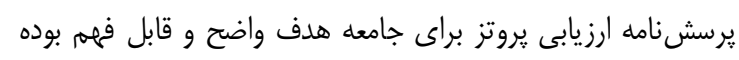

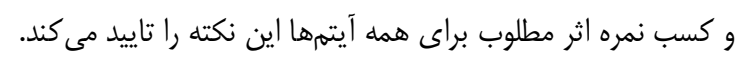

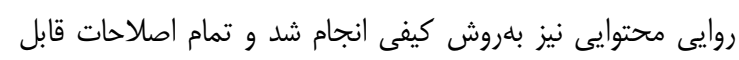

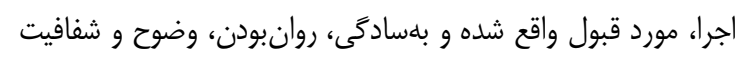

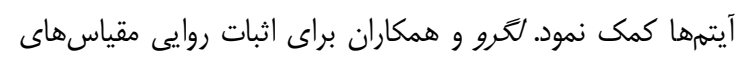

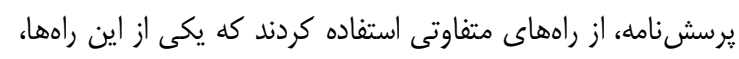

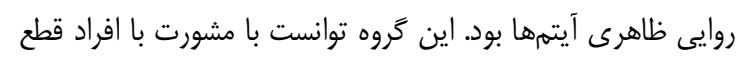

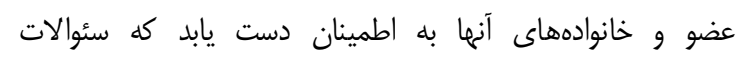

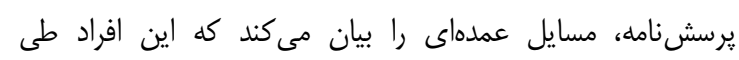

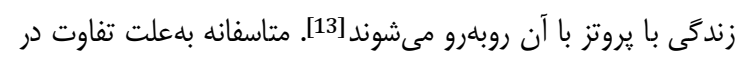

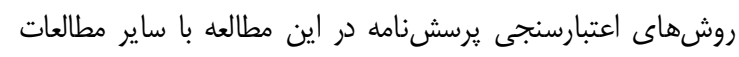

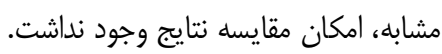

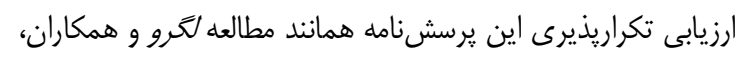

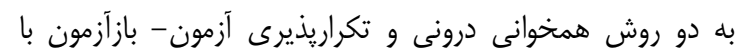

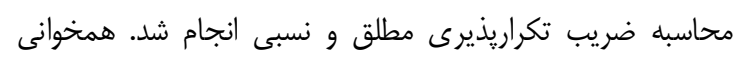

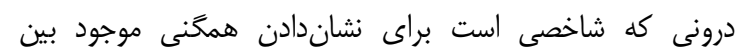

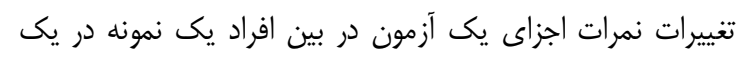

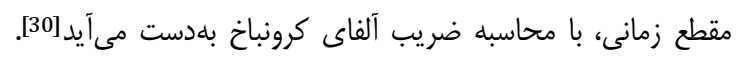

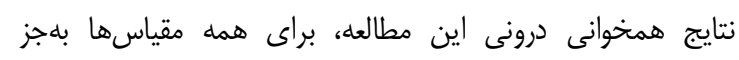

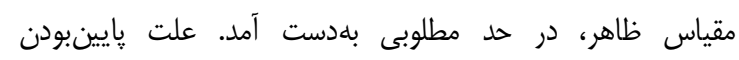

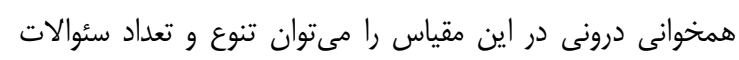

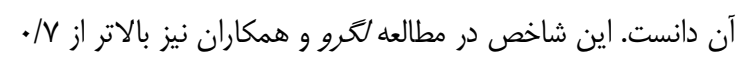

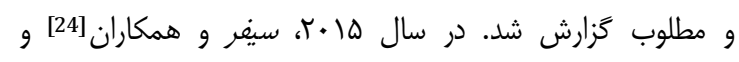

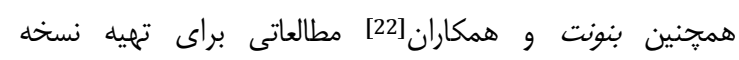

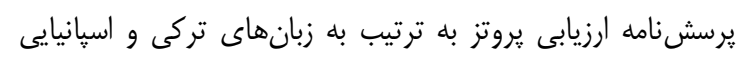

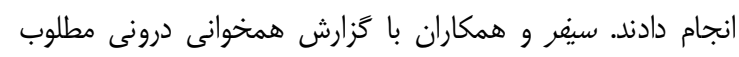

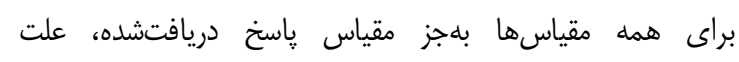

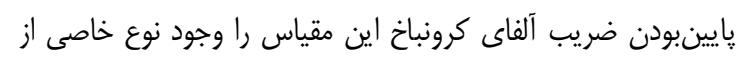

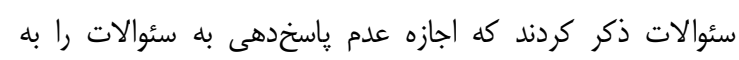

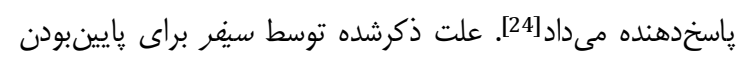

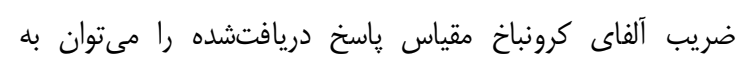

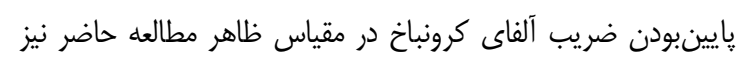
نسبت داد.

Scholarly Quarterly of Iranian Journal of War and Public Health
بود. روايى محتوايى برسشنامه نيز بلهصورت كيفى انجام شد و بعد أندات

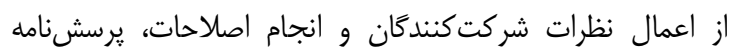
نهايى براى انجام مطالعه بلهدست آمد.

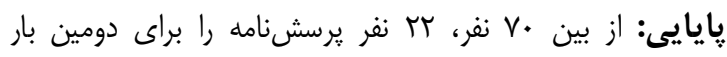

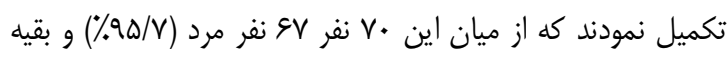

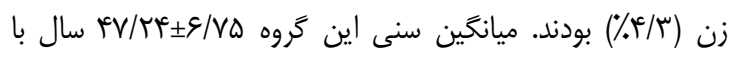

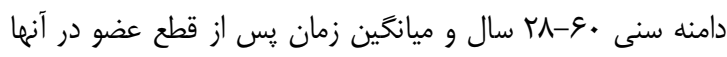

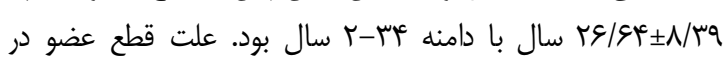

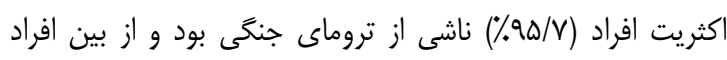

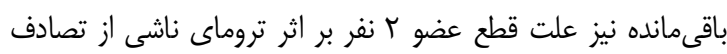
و يك نفر بر اثر بيمارى بود (جدول ()). جدول () فراوانى مطلق و نسبى جمعيتشناختى افراد داراى قطع عضو اندام

\begin{tabular}{|c|c|c|}
\hline درصد & فراوانى & مشخصات \\
\hline & & جنسيت \\
\hline$r / r$ & r & 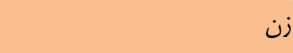 \\
\hline \multirow[t]{2}{*}{$90 / V$} & SV & مرد \\
\hline & & وضعيت تاهل \\
\hline $11 / 5$ & $\wedge$ & مجرد \\
\hline \multirow[t]{2}{*}{ NN/ $/ 9$} & gr & 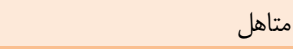 \\
\hline & & سطح تحصيلات \\
\hline $1 r / 9$ & 9 & زيردييلم \\
\hline rN/s & tr & دييلم \\
\hline$r E / r$ & IV & ليسانس \\
\hline \multirow[t]{2}{*}{$r E / r$} & iv & فوقليسانس، دكترا و بالاتر \\
\hline & & وضعيت شغلى \\
\hline$\Lambda F / r$ & DQ & 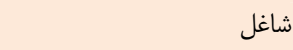 \\
\hline \multirow[t]{2}{*}{$\mid Q / V$} & 11 & بـ اكار \\
\hline & & توانايى رانندَى \\
\hline$\Lambda F / 1$ & $\Delta \wedge$ & داراى توانايى راندمَى \\
\hline \multirow[t]{2}{*}{$10 / 9$} & ir & عدم توانايى راندكىى \\
\hline & & سطح قطع عضو \\
\hline$r e / r$ & iv & بالاى زانو \\
\hline $1 . \%$ & v & روى زانو \\
\hline$\Delta I / F$ & 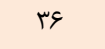 & زير زانو \\
\hline \multirow[t]{2}{*}{$\mid \varphi / r$} & 1. & قطع عضو مفصل مج \\
\hline & & علت قطع عضو \\
\hline $9 \mathrm{~N} / 9$ & 99 & تروما \\
\hline $1 / 4$ & 1 & بيمارى \\
\hline
\end{tabular}

ضريب تكراريذيرى نسبى (ICC) براى همه مقياسهاى

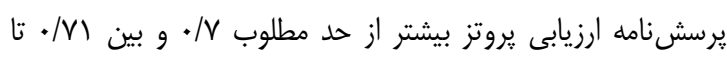

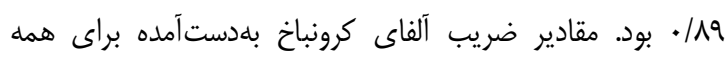

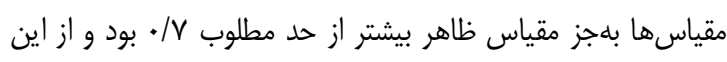

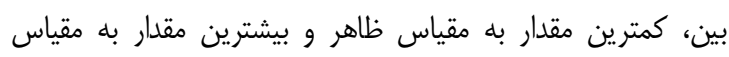

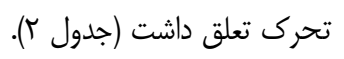




\begin{tabular}{|c|c|c|c|c|c|}
\hline ضريب آلفاى & 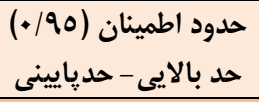 & ICC & دامنه & ميانَين آمارى & مقياسهاى يرسشنامه \\
\hline & & & & & تحرى (^ آيتم) \\
\hline \multirow[t]{2}{*}{.$/ 919$} & \multirow[t]{2}{*}{$.|8|-. / 94$} & \multirow[t]{2}{*}{$\cdot / \Lambda T^{2}$} & $\mid V / \Delta-1 \ldots$ & $\Delta F / N Q \pm T Y / G Y$ & مرحله اول \\
\hline & & & $\mid r / T V-q \vee / \wedge \Delta$ & $\Delta \Delta / r q \pm r \omega / r \wedge$ & مرحله دوم \\
\hline \multirow{3}{*}{. } & \multirow{3}{*}{$\cdot / 9 \cdot-\cdot / 94$} & \multirow{3}{*}{ 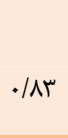 } & & & ظاهر (ه آيته) \\
\hline & & & $r T / \mathcal{F}-9 V$ & $\varepsilon r / 98 \pm \mid N / r$ & مرحله اول (•Vفر) \\
\hline & & & $r / / r-\wedge 9 / 1 r$ & $q \cdot / F \vee \pm 1 / / 99$ & مرحله دوم (rانفر) \\
\hline \multirow{3}{*}{$\cdot|A V|$} & & & & & صداها (ז آيتم) \\
\hline & \multirow{2}{*}{$\cdot / R T-\cdot / \mathrm{M}$} & \multirow{2}{*}{$\cdot / \mathrm{N} /$} & $\Lambda-q \vee / \Delta$ & $\varepsilon \cdot / V \pm r I / \& q$ & مرحله اول \\
\hline & & & $\mid r-1 .$. & $\Delta D / / Y \pm Y M / T \xi$ & مرحله دوم \\
\hline & \multirow{3}{*}{$. / 99-. / 97$} & \multirow{3}{*}{$\cdot / \Lambda 9$} & & & \multirow{3}{*}{ مرحله اولى عضل دور باقى مانده (ع آيتم) } \\
\hline \multirow{2}{*}{.$/ V I F$} & & & $r F-\wedge \varepsilon / \Delta$ & $\Delta r / \Delta \Lambda \pm 19 / \Delta T$ & \\
\hline & & & $r \cdot / \Lambda-V \Lambda / \wedge r$ & $\Delta \varepsilon / \Delta V \pm \mid N / \uparrow q$ & \\
\hline \multirow{3}{*}{.$/ A \& V$} & \multirow{3}{*}{$\cdot / V \Delta-\cdot / 9 \Delta$} & \multirow{3}{*}{.$/ 19$} & & & \multirow{2}{*}{ سودمندى (1 آيتم) } \\
\hline & & & $r T / V \Delta-q . / T \Delta$ & $\Delta \omega / Q \Lambda \pm r r / r q$ & \\
\hline & & & $r / / r V-q \Psi / \Lambda$ & $\Delta \Delta / \& \Delta \pm r \cdot / q \varnothing$ & مرحله دوم \\
\hline \multirow{3}{*}{.$/ \mathrm{Qq}$} & \multirow{3}{*}{$\cdot / \mathrm{TI}-\cdot / \mathrm{AM}$} & \multirow{3}{*}{$\cdot / \mathrm{NI}$} & & & ياسخ دريافتشده (צ آيتم) \\
\hline & & & $|f| / f-1 \ldots$ & $V D / G Y^{c} \pm I V / V G$ & مرحله اول \\
\hline & & & $r V / \Lambda-1 .$. & $V \varepsilon / r r \pm r \cdot|q|$ & 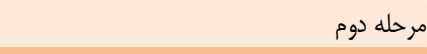 \\
\hline \multirow{3}{*}{.$/ V T A$} & \multirow{3}{*}{$\cdot / 90-\cdot / 9)^{C}$} & \multirow{3}{*}{$\cdot / \Lambda \Delta$} & & & بار اجتماعى (ץ آيتم) \\
\hline & & & $1 \cdot-1 .$. & $V \cdot / I I \pm T F / r \Lambda$ & مرحله اول \\
\hline & & & $r 1 / \Delta-1 .$. & $V r / \cdot \varepsilon \pm r I / 1 \varepsilon$ & مرحله دوم \\
\hline \multirow{3}{*}{.$/ 191$} & \multirow{3}{*}{$.|9|-\cdot / 94$} & \multirow{3}{*}{. / } & & & ناميدى (ץ آيته) \\
\hline & & & $\cdot-1+\cdot$ & $\Delta \varepsilon / q \pm M F / \cdot q$ & مرحله اول \\
\hline & & & $1 \cdot-9 V$ & $\Delta \Delta / q \pm \Gamma \cdot|\Delta|$ & 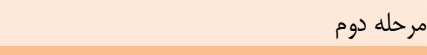 \\
\hline \multirow{3}{*}{.$/$ MY } & \multirow{3}{*}{ •/०१-•/१५ } & \multirow{3}{*}{ 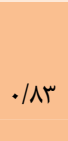 } & & & \multirow{3}{*}{ 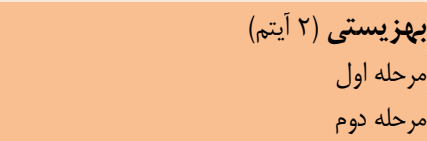 } \\
\hline & & & $N / \Delta-1 .$. & $\varepsilon \cdot / \Delta r \pm r g / M \Lambda$ & \\
\hline & & & $1 f / \Delta-9 V$ & $\Delta q / r r \pm r F / F q$ & \\
\hline
\end{tabular}

اين يثوهش در مقايسه با مطالعه لزرو و همكاران و ساير مطالعات

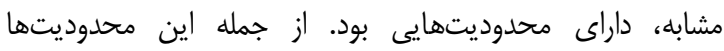

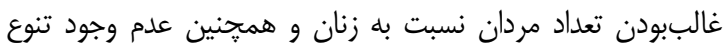

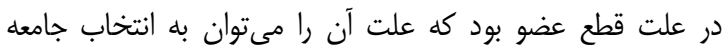

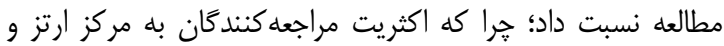
يروتز كوثر را جانبازان مرد تشكيل مىدهند.

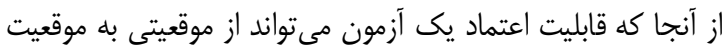

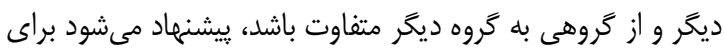

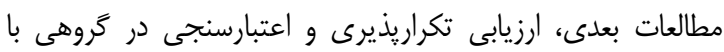
علل متفاوت قطع عضو و همجنين با توازن متعادل بين تعداد شركت كنندكان زن و مرد مورد بررسى قرار كيرد.

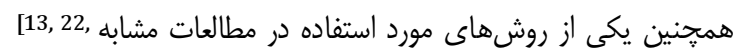
[24] استفاده از فرم كوتاهشده يروفايل وضعيت سلامتى (SF36) براى ارزيابى روايى بود. در اين مطالعات با بررسى همبستخى بين
بنونت و همكاران نيز نتايج اين شاخص را براى جهار مقياس در حد

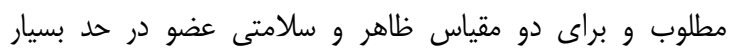

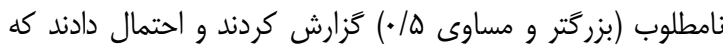

علت اين نامطلوبى، تعداد كم سئوالات اين مقياسها باشد [22].

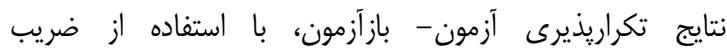

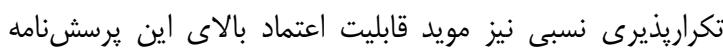

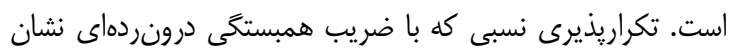

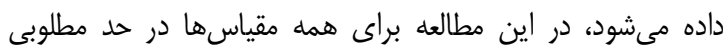

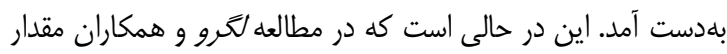
ICC

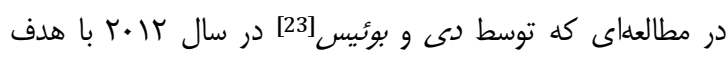
توليد نسخه عربى اين يرسشنامه انجام شد، اين شاخص براى همه

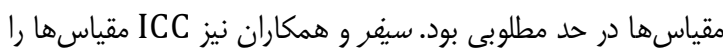

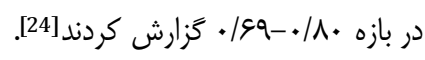




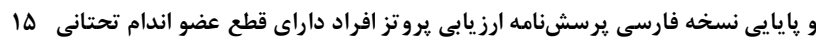

9- Pernot HF1, Winnubst GM, Cluitmans JJ, De Witte LP. Amputees in limburg: Incidence, morbidity and mortality, prosthetic supply, care utilisation and functional level after one year. Prosthet Orthot Int. 2000;24(2):90-6.

10- Prosthetics research study (PRS) [Internet]. Washington: Prosthetics research study; c2014-10 [Cited 2009, 12 February]. Available from: http://www.prsresearch.org/htmPages/PEQ.html.

11- Feinstein AR, Josephy BR, Wells CK. Scientific and clinical problems in indexes of functional disability. Ann Intern Med. 1986;105(3):413-20.

12- Keith RA. Functional assessment measures in medical rehabilitation: Current status. Arch Phys Med Rehab. 1984;65(2):74-8.

13- Legro MW, Reiber GD, Smith DG, del Aguila M, Larsen J, Boone D. Prosthesis evaluation questionnaire for persons with lower limb amputations: assessing prosthesis-related quality of life. Arch Phys Med Rehabil. 1998;79(8):931-8.

14- Condie E, Scott H, Treweek S. Lower limb prosthetic outcome measures: a review of the literature 1995 to 2005. J Prosthet Orthot. 2006;18(6):13-45.

15- Hawkins AT, Henry AJ, Crandell DM, Nguyen LL. A systematic review of functional and quality of life assessment after maj or lower extremity amputation. Ann Vasc Surg. 2014;28(3):763-780.

16- Boone DA, Coleman KL. Use of the prosthesis evaluation questionnaire (PEQ). J Prosthet Orthot. 2006;18(6):68-79.

17- Beaton DE, Bombardier C, Guillemin F, Ferraz MB. Guidelines for the process of cross-cultural adaptation of self-report measures. Spine. 2000;25(24):3186-91.

18- Tobimatsu Y, Iwaya T, Tamura T. Prosthesis-related QOL of the people with amputation in Japan. Hong Kong: $11^{\text {th }}$ World Congress of the International Society for Prosthetics \& Orthotics; 2004, August 1-6. p. 167.

19- Chu CK, Wong MS. Comparison of prosthetic outcomes between adolescent transtibial and transfemoral amputees after Sichuan earthquake using Step Activity Monitor and Prosthesis Evaluation Questionnaire. Prosthet Orthot Int. 2016;40(1):58-64.

20- Ferriero G, Dughi D, Orlandini D, Moscato T, Nicita D, Franchignoni F. Measuring long-term outcome in people with lower limb amputation: cross-validation of the Italian versions of the Prosthetic Profile of the Amputee and Prosthesis Evaluation Questionnaire. Eura Medicophys. 2005;41(1):1-6.

21- Franchignoni F, Giordano A, Ferriero G, Orlandini D, Amoresano A, Perucca L. Measuring mobility in people with lower limb amputation: Rasch analysis of the mobility section of the prosthesis evaluation questionnaire. J Rehabil Med. 2007;39(2):138-44.

22- Benavent JV, Igual C, Mora E, Antonio R, Tenias JM. Cross-cultural validation of the Prosthesis Evaluation Questionnaire in vascular amputees fitted with prostheses in Spain. Prosthet Orthot Int. 2015;1-7.

23- Day SJ, Buis A. Cross cultural equivalence testing of the Prosthetic Evaluation Questionnaire (PEQ) for an Arabic speaking population. Prosthet Orthot Int. 2012;36(2):173-80.

24- Safer VB, Yavuzer G, Demir So, Yanikoglu I, Guneri FD. The prosthesis evaluation questionnaire: Reliability and cross-validation of the Turkish version. J Phys Ther Sci. 2015;27(6):1677-80.

25- Bullinger M, Alonso J, Apolone G, Leplège A, Sullivan

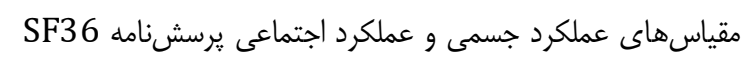

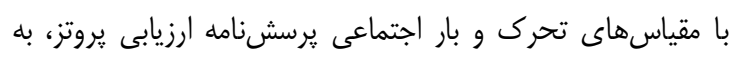

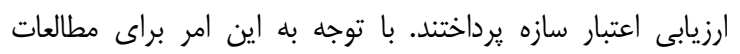

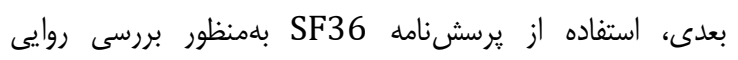

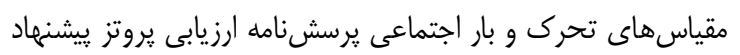
مىشود[31]. - مقئ.

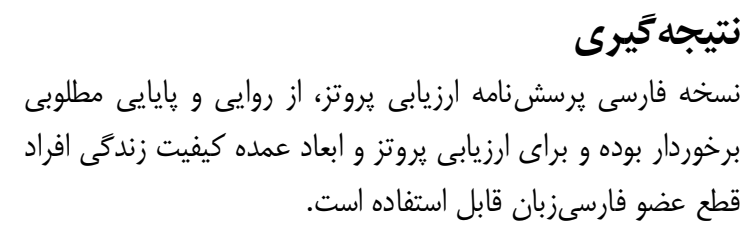
تشكر و قدردانى: با تقدير و تشكر فراوان از مديريت محترم و

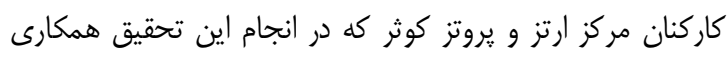
داشتند. تاييديه اخلاقى: اين يزوهش، از لحاظ اخلاقى توسط معاونت

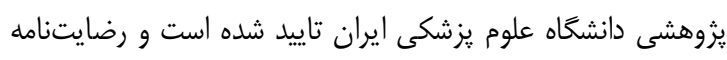

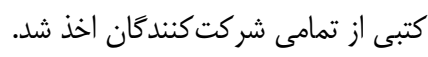

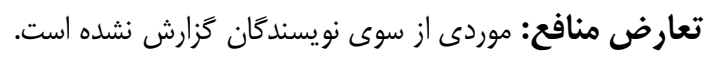

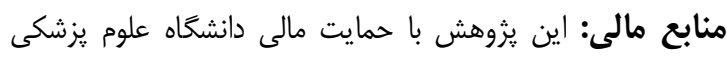

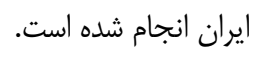

\section{منابع}

1- Bowker JH. Atlas of limb prosthetics: Surgical, prosthetic, and rehabilitation principles. 2nd edition. Mosby: Mosby-Year Book; 1992.

2- Devereux PG, Bullock CC, Bargmann-Losche J, Kyriakou M. Maintaining support in people with paralysis: What works? Qual Health Res. 2005;15(10):1360-76.

3- Lusardi MM, Jorge M, Nielsen CC. Orthotics and prosthetics in rehabilitation. 3rd edition. St. Louis: Elsevier Health Sciences; 2012.

4- Zargar M, Araghizadeh H, Soroush MR, Khaji A. Iranian casualties during the eight years of Iraq-Iran conflict. Rev Saude Publica. 2007;41(6):1065-6.

5- Fayers PM, Machin D. Quality of life: The assessment, analysis and interpretation of patient-reported outcomes. $2^{\text {nd }}$ edition. Chichester: John Wiley; 2007.

6- Hope ML, Page AC, Hooke GR. The value of adding the Quality of Life Enjoyment and Satisfaction Questionnaire to outcome assessments of psychiatric inpatients with mood and affective disorders. Qual Life Res. 2009;18(5):647-55.

7- Ostlie K, Franklin RJ, Skjeldal OH, Skrondal A, Magnus P.. Musculoskeletal pain and overuse syndromes in adult acquired major upper-limb amputees. Arch Phys Med Rehabil. 2011;92(12):1967-73.

8- Williams LH, Miller DR, Fincke G, Lafrance JP, Etzioni $\mathrm{R}$, Maynard C, et al. Depression and incident lower limb amputations in veterans with diabetes. J Diabetes Complicat. 2011;25(3):175-82. 
29- Santos JRA. Cronbach's alpha: A tool for assessing the reliability of scales. J Ext. 1999;37(2):1-5.

30- Finch E, Brooks D, Stratford P, Mayo N. How to choose outcomes relevant to the client and the rehabilitation program. Physical rehabilitation outcome measures. 2nd edition. Philadelphia: Lippincott Williams \& Wilkins; 2002. p. 6-15.

31- Montazeri A, Goshtasebi A, Vahdaninia M, Gandek B. The short form health survey (SF-36): Translation and validation study of the Iranian version. Qual Life Res. 2005;14(3):875-82.
M, Wood-Dauphinee $S$, et al. Translating health status questionnaires and evaluating their quality: The IQOLA project approach. J Clin Epidemiol. 1998;51(11):913-23. 26- Cohen L, Manion L, Morison K. Research Methods in Education. 7th edition. London: Routledge; 2011.

27- de Vet HC, Terwee C, Mokkink L, Knol D. Measurement in medicine: a practical guide. Cambridge: Cambridge University Press; 2011.

28- Weir JP. Quantifying test-retest reliability using the intraclass correlation coefficient and the SEM. J Strength Cond Res. 2005;19(1):231-40. 